CDAM RESEARCH REPORT LSE-CDAM-2007-38

\title{
A NEW APPROACH TO THE GIANT COMPONENT PROBLEM
}

\author{
SVANTE JANSON AND MALWINA J. LUCZAK
}

\begin{abstract}
We study the largest component of a random (multi)graph on $n$ vertices with a given degree sequence. We let $n \rightarrow \infty$. Then, under some regularity conditions on the degree sequences, we give conditions on the asymptotic shape of the degree sequence that imply that with high probability all the components are small, and other conditions that imply that with high probability there is a giant component and the sizes of its vertex and edge sets satisfy a law of large numbers; under suitable assumptions these are the only two possibilities. In particular, we recover the results by Molloy and Reed [24; 25] on the size of the largest component in a random graph with a given degree sequence.

We further obtain a new sharp result for the giant component just above the threshold, generalizing the case of $G(n, p)$ with $n p=1+$ $\omega(n) n^{-1 / 3}$, where $\omega(n) \rightarrow \infty$ arbitrarily slowly.

Our method is based on the properties of empirical distributions of independent random variables, and leads to simple proofs.
\end{abstract}

\section{INTRODUCTION}

For many years, questions concerning the size and structure of the largest component in a random graph have attracted a lot of attention. There have by now been quite a number of studies for the Bernoulli random graph $G(n, p)$ with $n$ vertices and edge probability $p$, and for the uniformly random graph $G(n, m)$ with $n$ vertices and $m$ edges (see for instance $[5 ; 17]$ and the references therein). Further, a number of studies $[24 ; 25 ; 19]$ have considered the emergence of a giant component in a random graph with a specified degree sequence. In [24], Molloy and Reed found the threshold for the appearance of a giant component in a random graph on $n$ vertices with a given degree sequence; in [25], they gave further results including the size of this giant component above the critical window. Their strategy was to analyse an edge deletion algorithm that finds the components in a graph, showing that the corresponding random process is well approximated by the solution to a system of differential equations. The proof is rather long and complicated, and uses a bound of the order $n^{1 / 4}$ on the maximum degree. More recently, Kang and Seierstad [19] have considered the near-critical behaviour of such graphs, once again assuming that, for some $\epsilon>0$, the

Date: 12 July 2007; revised 25 January 2008.

2000 Mathematics Subject Classification. 05C80; 60C05.

Key words and phrases. random graph, giant component, death process, empirical distribution. 
maximum degree does not exceed $n^{1 / 4-\epsilon}$. Using singularity analysis of generating functions, they determine the size of the giant component very close to the critical window, with a gap logarithmic in the number of vertices.

In this paper, we present a simple solution to the giant component problem. Unlike Molloy and Reed [24, 25], we do not use differential equations, but rely solely on the convergence of empirical distributions of independent random variables. (We use a variant of the method we used in $[15 ; 16]$ to study the $k$-core of a random graph.) In the super-critical regime, we require only conditions on the second moment of the asymptotic degree distribution; in the critical regime, we require a fourth moment condition, but we are able to go all the way to the critical window (at least for some degree sequences, see Remark 3.1), without any logarithmic separation. This is striking, as that logarithmic (or even larger) separation is often very hard to get rid of, see for instance [12] in the case of percolation on the Hamming graph $H(2, n)$ (i.e. the Cartesian product of two complete graphs on $n$ vertices), or [7] in percolation on the $n$-cube, and also [19] for the model analysed in the present paper. Like Molloy and Reed [24, 25], we work directly in the configuration model used to construct the random graph, exposing the edges one by one as they are needed.

We work with random graphs with given vertex degrees. Results for some other random graph models, notably for $G(n, p)$ and $G(n, m)$, follow immediately by conditioning on the vertex degrees.

Our method uses a version of the standard exploration of components. A commonly used, and very successful, method to study the giant component is to make a branching process approximation of the early stages of this exploration, thus focussing on the beginning of the exploration of each component and the conditions for not becoming extinct too soon; see e.g. Janson, Łuczak and Ruciński [17]; Molloy and Reed [24]; Kang and Seierstad [19] and, for some more complicated cases, Britton, Janson and Martin-Löf [8]. It should be noted that, in contrast, our method focuses on the condition for ending each exploration. The main idea of the method is to use a version of the exploration process where some half-edges are labelled as active, and where it is easy to find the asymptotics of the number of active half-edges, and thus to find when it becomes 0 , signifying the end of the exploration of the first component, see Sections 4 and 5.

\section{Notation AND RESUlts}

To state our results we introduce some notation. For a graph $G$, let $v(G)$ and $e(G)$ denote the numbers of vertices and edges in $G$, respectively; further, let $v_{k}(G)$ be the number of vertices of degree $k, k \geq 0$.

Let $n \in \mathbb{N}$ and let $\left(d_{i}\right)_{1}^{n}$ be a sequence of non-negative integers. We let $G\left(n,\left(d_{i}\right)_{1}^{n}\right)$ be a random graph with degree sequence $\left(d_{i}\right)_{1}^{n}$, uniformly chosen among all possibilities (tacitly assuming that there is any such graph at all). 
It will be convenient in the proofs below to work with multigraphs, that is to allow multiple edges and loops. More precisely, we shall use the following standard type of random multigraph: Let $n \in \mathbb{N}$ and let $\left(d_{i}\right)_{1}^{n}$ be a sequence of non-negative integers such that $\sum_{i=1}^{n} d_{i}$ is even. We let $G^{*}\left(n,\left(d_{i}\right)_{1}^{n}\right)$ be the random multigraph with given degree sequence $\left(d_{i}\right)_{1}^{n}$, defined by the configuration model (see e.g. Bollobás [5]): take a set of $d_{i}$ half-edges for each vertex $i$, and combine the half-edges into pairs by a uniformly random matching of the set of all half-edges. Note that $G^{*}\left(n,\left(d_{i}\right)_{1}^{n}\right)$ does not have exactly the uniform distribution over all multigraphs with the given degree sequence; there is a weight with a factor $1 / j$ ! for every edge of multiplicity $j$, and a factor $1 / 2$ for every loop, see e.g. [14, §1]. However, conditioned on the multigraph being a (simple) graph, we obtain $G\left(n,\left(d_{i}\right)_{1}^{n}\right)$, the uniformly distributed random graph with the given degree sequence.

We assume throughout the paper that we are given a sequence $\left(d_{i}\right)_{1}^{n}=$ $\left(d_{i}^{(n)}\right)_{1}^{n}$ for each $n \in \mathbb{N}$ (or at least for some sequence $n \rightarrow \infty$ ); for notational simplicity we will usually not show the dependence on $n$ explicitly. We consider asymptotics as $n \rightarrow \infty$, and all unspecified limits below are as $n \rightarrow \infty$. We say that an event holds whp (with high probability), if it holds with probability tending to 1 as $n \rightarrow \infty$. We shall use $\stackrel{\mathrm{p}}{\longrightarrow}$ for convergence in probability and $O_{\mathrm{p}}$ and $o_{\mathrm{p}}$ in the standard way (see e.g. Janson, Euczak and Ruciński [17]); for example, if $\left(X_{n}\right)$ is a sequence of random variables, then $X_{n}=O_{\mathrm{p}}(1)$ means " $X_{n}$ is bounded in probability" and $X_{n}=o_{\mathrm{p}}(1)$ means that $X_{n} \stackrel{\mathrm{p}}{\longrightarrow} 0$.

We write

$$
m=m(n):=\frac{1}{2} \sum_{i=1}^{n} d_{i}
$$

and

$$
n_{k}=n_{k}(n):=\#\left\{i: d_{i}=k\right\}, \quad k \geq 0
$$

thus $m$ is the number of edges and $n_{k}$ is the number of vertices of degree $k$ in the random graph $G\left(n,\left(d_{i}\right)_{1}^{n}\right)$ (or $G^{*}\left(n,\left(d_{i}\right)_{1}^{n}\right)$ ). We assume that the given $\left(d_{i}\right)_{1}^{n}$ satisfy the following regularity conditions, cf. Molloy and Reed $[24 ; 25]$ (where similar but not identical conditions are assumed).

Condition 2.1. For each $n,\left(d_{i}\right)_{1}^{n}=\left(d_{i}^{(n)}\right)_{1}^{n}$ is a sequence of non-negative integers such that $\sum_{i=1}^{n} d_{i}$ is even. Furthermore, $\left(p_{k}\right)_{k=0}^{\infty}$ is a probability distribution independent of $n$ such that

(i) $n_{k} / n=\#\left\{i: d_{i}=k\right\} / n \rightarrow p_{k}$ as $n \rightarrow \infty$, for every $k \geq 0$;

(ii) $\lambda:=\sum_{k} k p_{k} \in(0, \infty)$;

(iii) $\sum_{i} d_{i}^{2}=O(n)$;

(iv) $p_{1}>0$. 
Let $D_{n}$ be a random variable defined as the degree of a random (uniformly chosen) vertex in $G\left(n,\left(d_{i}\right)_{1}^{n}\right)$ or $G^{*}\left(n,\left(d_{i}\right)_{1}^{n}\right)$; thus

$$
\mathbb{P}\left(D_{n}=k\right)=n_{k} / n \text {. }
$$

Note that $\mathbb{E} D_{n}=n^{-1} \sum_{i=1}^{n} d_{i}=2 m / n$.

Further, let $D$ be a random variable with the distribution $\mathbb{P}(D=k)=p_{k}$. Then (i) can be written

$$
D_{n} \stackrel{\mathrm{d}}{\longrightarrow} D
$$

In other words, $D$ describes the asymptotic distribution of the degree of a random vertex in $G\left(n,\left(d_{i}\right)_{1}^{n}\right)$. Furthermore, (ii) is $\lambda=\mathbb{E} D \in(0, \infty)$, (iv) is $\mathbb{P}(D=1)>0$, and (iii) can be written

$$
\mathbb{E} D_{n}^{2}=O(1)
$$

Remark 2.2. In particular, (2.3) implies that the random variables $D_{n}$ are uniformly integrable, and thus Condition 2.1(i), in the form (2.2), implies $\mathbb{E} D_{n} \rightarrow \mathbb{E} D$, i.e.

$$
\frac{2 m}{n}=n^{-1} \sum_{i=1}^{n} d_{i} \rightarrow \lambda
$$

see e.g. [11, Theorems 5.4.2 and 5.5.9].

Let

$$
g(x):=\sum_{k=0}^{\infty} p_{k} x^{k}=\mathbb{E} x^{D},
$$

the probability generating function of the probability distribution $\left(p_{k}\right)_{k=0}^{\infty}$, and define further

$$
\begin{aligned}
& h(x):=x g^{\prime}(x)=\sum_{k=1}^{\infty} k p_{k} x^{k}, \\
& H(x):=\lambda x^{2}-h(x) .
\end{aligned}
$$

Note that $h(0)=0$ and $h(1)=\lambda$, and thus $H(0)=H(1)=0$. Note also that

$$
H^{\prime}(1)=2 \lambda-\sum_{k} k^{2} p_{k}=\mathbb{E}\left(2 D-D^{2}\right)=-\mathbb{E} D(D-2) .
$$

See further Lemma 5.5. $25]$.

Our first theorem is essentially the main results of Molloy and Reed [24,

Theorem 2.3. Suppose that Condition 2.1 holds and consider the random graph $G\left(n,\left(d_{i}\right)_{1}^{n}\right)$, letting $n \rightarrow \infty$. Let $\mathcal{C}_{1}$ and $\mathcal{C}_{2}$ be the largest and second largest components of $G\left(n,\left(d_{i}\right)_{1}^{n}\right)$.

(i) If $\mathbb{E} D(D-2)=\sum_{k} k(k-2) p_{k}>0$, then there is a unique $\xi \in(0,1)$ such that $H(\xi)=0$, or equivalently $g^{\prime}(\xi)=\lambda \xi$, and

$$
v\left(\mathcal{C}_{1}\right) / n \stackrel{\mathrm{p}}{\longrightarrow} 1-g(\xi)>0
$$




$$
\begin{aligned}
& v_{k}\left(\mathcal{C}_{1}\right) / n \stackrel{\mathrm{p}}{\longrightarrow} p_{k}\left(1-\xi^{k}\right), \text { for every } k \geq 0, \\
& e\left(\mathcal{C}_{1}\right) / n \stackrel{\mathrm{p}}{\longrightarrow} \frac{1}{2} \lambda\left(1-\xi^{2}\right),
\end{aligned}
$$

while $v\left(\mathcal{C}_{2}\right) / n \stackrel{\mathrm{p}}{\longrightarrow} 0$ and $e\left(\mathcal{C}_{2}\right) / n \stackrel{\mathrm{p}}{\longrightarrow} 0$.

(ii) If $\mathbb{E} D(D-2)=\sum_{k} k(k-2) p_{k} \leq 0$, then $v\left(\mathcal{C}_{1}\right) / n \stackrel{\mathrm{p}}{\longrightarrow} 0$ and $e\left(\mathcal{C}_{1}\right) / n \stackrel{\mathrm{p}}{\longrightarrow} 0$.

The same results hold for $G^{*}\left(n,\left(d_{i}\right)_{1}^{n}\right)$.

In the usual, somewhat informal, language, the theorem shows that $G\left(n,\left(d_{i}\right)_{1}^{n}\right)$ has a giant component if and only if $\mathbb{E} D(D-2)>0$.

In the critical case, we can be more precise.

Theorem 2.4. Suppose that Condition 2.1 holds and that $\mathbb{E} D(D-2)=$ $\sum_{k} k(k-2) p_{k}=0$. Assume further that $\alpha_{n}:=\mathbb{E} D_{n}\left(D_{n}-2\right)=\sum_{i=1}^{n} d_{i}\left(d_{i}-\right.$ $2) / n>0$ and, moreover, $n^{1 / 3} \alpha_{n} \rightarrow \infty$, and that

$$
\sum_{i=1}^{n} d_{i}^{4+\eta}=O(n)
$$

for some $\eta>0$. Let $\beta:=\mathbb{E} D(D-1)(D-2)$. Then, $\beta>0$ and

$$
\begin{aligned}
v\left(\mathcal{C}_{1}\right) & =\frac{2 \lambda}{\beta} n \alpha_{n}+o_{\mathrm{p}}\left(n \alpha_{n}\right), \\
v_{k}\left(\mathcal{C}_{1}\right) & =\frac{2}{\beta} k p_{k} n \alpha_{n}+o_{\mathrm{p}}\left(n \alpha_{n}\right), \text { for every } k \geq 0, \\
e\left(\mathcal{C}_{1}\right) & =\frac{2 \lambda}{\beta} n \alpha_{n}+o_{\mathrm{p}}\left(n \alpha_{n}\right),
\end{aligned}
$$

while $v\left(\mathcal{C}_{2}\right)=o_{\mathrm{p}}\left(n \alpha_{n}\right)$ and $e\left(\mathcal{C}_{2}\right) / n=o_{\mathrm{p}}\left(n \alpha_{n}\right)$.

The same results hold for $G^{*}\left(n,\left(d_{i}\right)_{1}^{n}\right)$.

Remark 2.5. Condition (2.9) may be written $\mathbb{E} D_{n}^{4+\eta}<\infty$; it thus implies (2.3) and Condition 2.1(iii); moreover, it implies that $D_{n}^{2}$ and $D_{n}^{3}$ are uniformly integrable. Hence, using (2.2), (2.9) implies $\mathbb{E} D_{n}^{2} \rightarrow \mathbb{E} D^{2}$ and $\mathbb{E} D_{n}^{3} \rightarrow \mathbb{E} D^{3}$. In particular, the conditions of Theorem 2.4 imply

$$
\alpha_{n}:=\mathbb{E} D_{n}\left(D_{n}-2\right) \rightarrow \mathbb{E} D(D-2)=0
$$

and

$$
\beta_{n}:=\mathbb{E} D_{n}\left(D_{n}-1\right)\left(D_{n}-2\right) \rightarrow \mathbb{E} D(D-1)(D-2)=\beta .
$$

We do not think that the condition (2.9) is best possible; we conjecture that, in addition to Condition 2.1, it is enough to assume that $D_{n}^{3}$ are uniformly integrable, or, equivalently, that $\mathbb{E} D_{n}^{3} \rightarrow \mathbb{E} D^{3}<\infty$.

Condition 2.1(iii) and (2.4) imply that

$$
\liminf \mathbb{P}\left(G^{*}\left(n,\left(d_{i}\right)_{1}^{n}\right) \text { is a simple graph }\right)>0,
$$

see for instance Bollobás [5], McKay [22] or McKay and Wormald [23] under some extra condition on $\max d_{i}$ and Janson [13] for the general case. Since 
we obtain $G\left(n,\left(d_{i}\right)_{1}^{n}\right)$ by conditioning $G^{*}\left(n,\left(d_{i}\right)_{1}^{n}\right)$ on being a simple graph, and all results in Theorems 2.3 and 2.4 are (or can be) stated in terms of convergence in probability, the results for $G\left(n,\left(d_{i}\right)_{1}^{n}\right)$ follow from the results for $G^{*}\left(n,\left(d_{i}\right)_{1}^{n}\right)$ by this conditioning.

We will prove Theorems 2.3 and 2.4 for $G^{*}\left(n,\left(d_{i}\right)_{1}^{n}\right)$ in Sections 5 and 6 . The proofs use the same arguments, but we find it convenient to first discuss the somewhat simpler case of Theorem 2.3 in detail and then do the necessary modifications for Theorem 2.4.

Remark 2.6. The assumption Condition 2.1(iii) is used in our proof mainly for the reduction to $G^{*}\left(n,\left(d_{i}\right)_{1}^{n}\right)$. In fact, the proof of Theorem 2.3 for $G^{*}\left(n,\left(d_{i}\right)_{1}^{n}\right)$ holds with simple modifications also if Condition 2.1(iii) is replaced by the weaker condition that $D_{n}$ are uniformly integrable, or equivalently, see Remark 2.2, $\mathbb{E} D_{n} \rightarrow \mathbb{E} D$ or (2.4). It might also be possible to extend Theorem 2.3 for $G\left(n,\left(d_{i}\right)_{1}^{n}\right)$ too, under some weaker assumption than Condition 2.1(iii), by combining estimates of $\mathbb{P}\left(G^{*}\left(n,\left(d_{i}\right)_{1}^{n}\right)\right.$ is simple) from e.g. McKay and Wormald [23] with more precise estimates of the error probabilities in Section 5, but we have not pursued this.

Remark 2.7. Condition 2.1(iv) excludes the case $p_{1}=0$; we comment briefly on this case here. Note first that in this case, $\mathbb{E} D(D-2)=\sum_{k=3}^{\infty} k(k-$ 2) $p_{k} \geq 0$, with strict inequality as soon as $p_{k}>0$ for some $k \geq 3$.

First, if $p_{1}=0$ and $\mathbb{E} D(D-2)>0$, i.e. if $p_{1}=0$ and $\sum_{k \geq 3} p_{k}>0$, it is easily seen (by modifying the proof of Theorem 2.3 below or by adding $\varepsilon n$ vertices of degree 1 and applying Theorem 2.3) that all but $o_{\mathrm{p}}(n)$ vertices and edges belong to a single giant component. Hence, the conclusions of Theorem 2.3(i) hold with $\xi=0$. (In this case, $H(x)>0$ for every $x \in(0,1)$.)

The case $p_{1}=0$ and $\mathbb{E} D(D-2)=0$, i.e. $p_{k}=0$ for all $k \neq 0,2$, is much more exceptional. (In this case, $H(x)=0$ for all $x$.) We give three examples showing that quite different behaviours are possible. Since isolated vertices do not matter, let us assume $p_{0}=0$ too and consider thus the case $p_{2}=1$.

One example is when all $d_{i}=2$, so we are studying a random 2-regular graph. In this case, the components are cycles. It is well-known, and easy to see, that (for the multigraph version) the distribution of cycle lengths is given by the Ewens's sampling formula $\operatorname{ESF}(1 / 2)$, see e.g. Arratia, Barbour and Tavaré [2], and thus $v\left(\mathcal{C}_{1}\right) / n$ converges in distribution to a non-degenerate distribution on $[0,1]$ and not to any constant [2, Lemma 5.7]. Moreover, the same is true for $v\left(\mathcal{C}_{2}\right) / n$ (and for $v\left(\mathcal{C}_{3}\right) / n, \ldots$ ), so in this case there are several large components.

A second case with $p_{2}=1$ is obtained by adding a small number of vertices of degree 1. (More precisely, let $n_{1} \rightarrow \infty, n_{1} / n \rightarrow 0$, and $n_{2}=n-n_{1}$.) It is then easy to see that $v\left(\mathcal{C}_{1}\right)=o_{\mathrm{p}}(n)$.

A third case with $p_{2}=1$ is obtained by instead adding a small number of vertices of degree 4 (i.e., $n_{4} \rightarrow \infty, n_{4} / n \rightarrow 0$, and $n_{2}=n-n_{4}$ ). By regarding each vertex of degree 4 as two vertices of degree 2 that have merged, it is 
easy to see that in this case $v\left(\mathcal{C}_{1}\right)=n-o_{\mathrm{p}}(n)$, so there is a giant component containing almost everything. (The case $\xi=0$ again.)

\section{3. $G(n, p), G(n, m)$ AND OTHER RANDOM GRAPHS}

The results above can be applied to some other random graph models too by conditioning on the vertex degrees; this works whenever the random graph conditioned on the degree sequence has a uniform distribution over all possibilities. Notable examples of such random graphs are $G(n, p)$ and $G(n, m)$, and other examples are given in [9], [6, Section 16.4], [10]. If, furthermore, Condition 2.1 and (2.9) hold in probability (where now $d_{i}$ are the random vertex degrees), then Theorems 2.3 and 2.4 hold; in the latter, we define $\alpha_{n}:=\sum_{i=1}^{n} d_{i}\left(d_{i}-2\right) / n$, which now is random. (For the proof, it is convenient to use the Skorohod coupling theorem [18, Theorem 4.30] and assume that the conditions hold a.s.)

For example, for $G(n, p)$ with $n p \rightarrow \lambda$ or $G(n, m)$ with $2 m / n \rightarrow \lambda$, where $0<\lambda<\infty$, the assumptions hold with $D \sim \operatorname{Po}(\lambda)$ and thus $g(x)=e^{\lambda(x-1)}$, $h(x)=\lambda x e^{\lambda(x-1)}, H(x)=\lambda x\left(x-e^{\lambda(x-1)}\right)$, and we recover both the classical threshold $\lambda=1$ and the standard equation $\xi=e^{\lambda(\xi-1)}$ for the size of the giant component when $\lambda>1$.

If we consider $G(n, p)$ with $p=\left(1+\varepsilon_{n}\right) / n$ where $\varepsilon_{n} \rightarrow 0$ in Theorem 2.4, we have $\alpha_{n} / \varepsilon_{n} \stackrel{\mathrm{p}}{\longrightarrow} 1$ by the second moment method as soon as $n \varepsilon_{n} \rightarrow \infty$, so we need $n^{1 / 3} \varepsilon_{n} \rightarrow \infty$ in order to apply Theorem 2.4. On the other hand, it is well known $[5 ; 14 ; 17]$ that if $n^{1 / 3} \varepsilon_{n}=O(1)$, then $v\left(\mathcal{C}_{1}\right)$ and $v\left(\mathcal{C}_{2}\right)$ are both of the same order $n^{2 / 3}$ and Theorem 2.4 fails, which shows that the condition $n^{1 / 3} \alpha_{n} \rightarrow \infty$ in Theorem 2.4 is best possible.

Remark 3.1. Consider again the critical case $\alpha_{n}:=\mathbb{E} D_{n}\left(D_{n}-2\right) \rightarrow 0$. Since Theorem 2.4 shows that then the largest component of $G\left(n,\left(d_{i}\right)_{1}^{n}\right)$ has the same general behaviour as for $G(n, p)$ for $\alpha_{n} \gg n^{-1 / 3}$, we conjecture by extrapolation that the same holds for $\alpha_{n}=O\left(n^{-1 / 3}\right)$ and $n^{1 / 3} \alpha_{n} \rightarrow-\infty$ too, i.e., that when $n^{1 / 3} \alpha_{n} \rightarrow \alpha \in \mathbb{R}$, then $v\left(\mathcal{C}_{1}\right)=O_{\mathrm{p}}\left(n^{2 / 3}\right)$ (presumably with a limiting distribution of $v\left(\mathcal{C}_{1}\right) / n^{2 / 3}$ but not a deterministic limit, and presumably with the second largest component of comparable order), and that when $n^{1 / 3} \alpha_{n} \rightarrow-\infty$, then $v\left(\mathcal{C}_{1}\right)=o_{\mathrm{p}}\left(n^{2 / 3}\right)$. In other words, we believe that $\alpha_{n}=O\left(n^{-1 / 3}\right)$ is the correct critical window. Apart from the case $G(n, p)$, which as just said is an example if we allow random degree sequences, such results have also recently been shown for the case of critical percolation in a random $d$-regular graph by Nachmias and Peres [26], which corresponds to the case $D \sim \operatorname{Bi}(d, 1 /(d-1))$. It would be interesting to show such results for general degree sequences. 


\section{Finding the Largest COMPonent}

The components of an arbitrary finite graph or multigraph can be found by the following standard procedure. Pick an arbitrary vertex $v$ and determine the component of $v$ as follows: include all the neighbours of $v$ in an arbitrary order; then add in the neighbours of neighbours, and so on, until no more vertices can be added. The vertices included until this moment form the component of $v$. If there are still vertices left in the graph, pick any such vertex $w$, and repeat the above to determine the second component (the component of vertex $w$ ). Carry on in this manner until all the components have been found.

It is clear that we obtain the same result as follows. Regard each edge as consisting of two half-edges, each half-edge having one endpoint. We will label the vertices as sleeping or awake (= used) and the half-edges as sleeping, active or dead; the sleeping and active half-edges are also called living. We start with all vertices and half-edges sleeping. Pick a vertex and label its half-edges as active. Then take any active half-edge, say $x$ and find its partner $y$ in the graph; label these two half-edges as dead; further, if the endpoint of $y$ is sleeping, label it as awake and all other half-edges there as active. Repeat as long as there is any active half-edge.

When there is no active half-edge left, we have obtained the first component. Then start again with another vertex until all components are found.

We apply this algorithm to a random multigraph $G^{*}\left(n,\left(d_{i}\right)_{1}^{n}\right)$ with a given degree sequence, revealing its edges during the process. We thus observe initially only the vertex degrees and the half-edges, but not how they are joined to form edges. Hence, each time we need a partner of an half-edge, it is uniformly distributed over all other living half-edges. (The dead half-edges are the ones that already are paired into edges.) We make these random choices by giving the half-edges i.i.d. random maximal lifetimes $\tau_{x}$ with the distribution $\operatorname{Exp}(1)$; in other words, each half-edge dies spontaneously with rate 1 (unless killed earlier). Each time we need to find the partner of a half-edge $x$, we then wait until the next living half-edge $\neq x$ dies and take that one. We then can formulate an algorithm, constructing $G^{*}\left(n,\left(d_{i}\right)_{1}^{n}\right)$ and exploring its components simultaneously, as follows. Recall that we start with all vertices and half-edges sleeping.

C1 If there is no active half-edge (as in the beginning), select a sleeping vertex and declare it awake and all its half-edges active. For definiteness, we choose the vertex by choosing a half-edge uniformly at random among all sleeping half-edges. If there is no sleeping halfedge left, the process stops; the remaining sleeping vertices are all isolated and we have explored all other components.

C2 Pick an active half-edge (which one does not matter) and kill it, i.e., change its status to dead.

C3 Wait until the next half-edge dies (spontaneously). This half-edge is joined to the one killed in the previous step C2 to form an edge of the 
graph. If the vertex it belongs to is sleeping, we change this vertex to awake and all other half-edges at this vertex to active. Repeat from $\mathrm{C} 1$.

The components are created between the successive times $\mathrm{C} 1$ is performed; the vertices in the component created during one of these intervals are the vertices that are awakened during the interval. Note also that a component is completed and $\mathrm{C} 1$ is performed exactly when the number of active half-edges is 0 and in step C3 a half-edge dies at a vertex where all other half-edges (if any) already are dead.

\section{Analysis of the Algorithm for $G^{*}\left(n,\left(d_{i}\right)_{1}^{n}\right)$}

Let $S(t)$ and $A(t)$ be the numbers of sleeping and active half-edges, respectively, at time $t$, and let $L(t)=S(t)+A(t)$ be the number of living half-edges. As is customary, and for definiteness, we define these random functions to be right-continuous.

Let us first look at $L(t)$. We start with $2 m$ half-edges, all sleeping and thus living, but we immediately perform $\mathrm{C} 1$ and $\mathrm{C} 2$ and kill one of them; thus $L(0)=2 m-1$. In the sequel, as soon as a living half-edge dies, we perform C3 and then (instantly) either C2 or both C1 and C2. Since C1 does not change the number of living half-edges while C2 and C3 each decrease it by 1 , the total result is that $L(t)$ is decreased by 2 each time one of the living half-edges dies, except when the last living one dies and the process terminates.

Lemma 5.1. As $n \rightarrow \infty$,

$$
\sup _{t \geq 0}\left|n^{-1} L(t)-\lambda e^{-2 t}\right| \stackrel{\mathrm{p}}{\longrightarrow} 0 .
$$

Proof. This (or rather an equivalent statement in a slightly different situation) was proved in [15] as a consequence of the Glivenko-Cantelli theorem [18, Proposition 4.24] on convergence of empirical distribution functions. It also follows easily from (the proof of) Lemma 6.2 below if we replace $\alpha_{n}$ by 1.

Next consider the sleeping half-edges. Let $V_{k}(t)$ be the number of sleeping vertices of degree $k$ at time $t$; thus

$$
S(t)=\sum_{k=1}^{\infty} k V_{k}(t)
$$

Note that C2 does not affect sleeping half-edges, and that C3 implies that each sleeping vertex of degree $k$ is eliminated (i.e., awakened) with intensity $k$, independently of all other vertices. There are also some sleeping vertices eliminated by $\mathrm{C} 1$.

We first ignore the effect of $\mathrm{C} 1$ by letting $\widetilde{V}_{k}(t)$ be the number of vertices of degree $k$ such that all its half-edges have maximal lifetimes $\tau_{x}>t$. (I.e., none of its $k$ half-edges would have died spontaneously up to time $t$, assuming 
they all escaped C1.) Let further $\widetilde{S}(t):=\sum_{k} k \widetilde{V}_{k}(t)$. Recall the functions $g, h$ from (2.5), (2.6).

Lemma 5.2. As $n \rightarrow \infty$,

$$
\sup _{t \geq 0}\left|n^{-1} \widetilde{V}_{k}(t)-p_{k} e^{-k t}\right| \stackrel{\mathrm{p}}{\longrightarrow} 0
$$

for every $k \geq 0$ and

$$
\begin{gathered}
\sup _{t \geq 0}\left|n^{-1} \sum_{k=0}^{\infty} \widetilde{V}_{k}(t)-g\left(e^{-t}\right)\right| \stackrel{\mathrm{p}}{\longrightarrow} 0, \\
\quad \sup _{t \geq 0}\left|n^{-1} \widetilde{S}(t)-h\left(e^{-t}\right)\right| \stackrel{\mathrm{p}}{\longrightarrow} 0 .
\end{gathered}
$$

Proof. The statement (5.1), again, follows from the Glivenko-Cantelli theorem, see [15], or from the proof of Lemma 6.3 below. (The case $k=0$ is trivial, with $\widetilde{V}_{0}(t)=n_{0}$ for all $t$.)

By Remark 2.2, $D_{n}$ are uniformly integrable, which means that for every $\varepsilon>0$ there exists $K<\infty$ such that for all $n, \sum_{k>K} k n_{k} / n=\mathbb{E}\left(D_{n} ; D_{n}>\right.$ $K)<\varepsilon$. We may further assume (or deduce by Fatou's inequality) $\sum_{k>K} k p_{k}<$ $\varepsilon$, and obtain by (5.1) whp

$$
\begin{aligned}
\sup _{t \geq 0} \mid n^{-1} \widetilde{S}(t) & -h\left(e^{-t}\right)\left|=\sup _{t \geq 0}\right| \sum_{k=1}^{\infty} k\left(n^{-1} \widetilde{V}_{k}(t)-p_{k} e^{-k t}\right) \mid \\
& \leq \sum_{k=1}^{K} k \sup _{t \geq 0}\left|n^{-1} \widetilde{V}_{k}(t)-p_{k} e^{-k t}\right|+\sum_{k>K} k\left(\frac{n_{k}}{n}+p_{k}\right) \\
& \leq \varepsilon+\varepsilon+\varepsilon,
\end{aligned}
$$

proving (5.3). An almost identical argument yields (5.2).

The difference between $S(t)$ and $\widetilde{S}(t)$ is easily estimated.

Lemma 5.3. If $d_{\max }:=\max _{i} d_{i}$ is the maximum degree of $G^{*}\left(n,\left(d_{i}\right)_{1}^{n}\right)$, then

$$
0 \leq \widetilde{S}(t)-S(t)<\sup _{0 \leq s \leq t}(\widetilde{S}(s)-L(s))+d_{\max } .
$$

Proof. Clearly, $V_{k}(t) \leq \widetilde{V}_{k}(t)$, and thus $S(t) \leq \widetilde{S}(t)$; furthermore, $\widetilde{S}(t)-S(t)$ increases only as a result of $\mathrm{C} 1$, which acts to guarantee that $A(t)=L(t)-$ $S(t) \geq 0$.

If $\mathrm{C} 1$ is performed at time $t$ and a vertex of degree $j>0$ is awakened, then C2 applies instantly and we have $A(t)=j-1<d_{\max }$, and consequently

$$
\widetilde{S}(t)-S(t)=\widetilde{S}(t)-L(t)+A(t)<\widetilde{S}(t)-L(t)+d_{\max } .
$$

Furthermore, $\widetilde{S}(t)-S(t)$ is never changed by $\mathrm{C} 2$ and either unchanged or decreased by C3. Hence, $\widetilde{S}(t)-S(t)$ does not increase until the next time $\mathrm{C} 1$ is performed. Consequently, for any time $t$, if $s$ was the last time before 
(or equal to) $t$ that $\mathrm{C} 1$ was performed, then $\widetilde{S}(t)-S(t) \leq \widetilde{S}(s)-S(s)$, and the result follows by (5.4).

Let

$$
\widetilde{A}(t):=L(t)-\widetilde{S}(t)=A(t)-(\widetilde{S}(t)-S(t)) .
$$

Then, by Lemmas 5.1 and 5.2 and (2.7),

$$
\sup _{t \geq 0}\left|n^{-1} \widetilde{A}(t)-H\left(e^{-t}\right)\right| \stackrel{\mathrm{p}}{\longrightarrow} 0 .
$$

Lemma 5.3 can be written

$$
0 \leq \widetilde{S}(t)-S(t)<-\inf _{s \leq t} \widetilde{A}(s)+d_{\max }
$$

Remark 5.4. By (5.5) and (5.7), we obtain further the relation

$$
\widetilde{A}(t) \leq A(t)<\widetilde{A}(t)-\inf _{s \leq t} \widetilde{A}(s)+d_{\max }
$$

which, perhaps, illuminates the relation between $A(t)$ and $\widetilde{A}(t)$.

Lemma 5.5. Suppose that Condition 2.1 holds and let $H(x)$ be given by (2.7).

(i) If $\mathbb{E} D(D-2)=\sum_{k} k(k-2) p_{k}>0$, then there is a unique $\xi \in(0,1)$ such that $H(\xi)=0$; moreover, $H(x)<0$ for $x \in(0, \xi)$ and $H(x)>0$ for $x \in(\xi, 1)$.

(ii) If $\mathbb{E} D(D-2)=\sum_{k} k(k-2) p_{k} \leq 0$, then $H(x)<0$ for $x \in(0,1)$.

Proof. As remarked earlier, $H(0)=H(1)=0$ and $H^{\prime}(1)=-\mathbb{E} D(D-2)$. Furthermore, if we define $\varphi(x):=H(x) / x$, then $\varphi(x)=\lambda x-\sum_{k} k p_{k} x^{k-1}$ is a concave function on $(0,1]$, and it is strictly concave unless $p_{k}=0$ for all $k \geq 3$, in which case $H^{\prime}(1)=-\mathbb{E} D(D-2)=p_{1}>0$.

In case (ii), we thus have $\varphi$ concave and $\varphi^{\prime}(1)=H^{\prime}(1)-H(1) \geq 0$, with either the concavity or the inequality strict, and thus $\varphi^{\prime}(x)>0$ for all $x \in(0,1)$, whence $\varphi(x)<\varphi(1)=0$ for $x \in(0,1)$.

In case (i), $H^{\prime}(1)<0$, and thus $H(x)>0$ for $x$ close to 1 . Further, $H^{\prime}(0)=-h^{\prime}(0)=-p_{1}<0$, and thus $H(x)<0$ for $x$ close to 0 . Hence there is at least one $\xi \in(0,1)$ with $H(\xi)=0$, and since $H(x) / x$ is strictly concave and also $H(1)=0$, there is at most one such $\xi$ and the result follows.

Proof of Theorem 2.3(i). Let $\xi$ be the zero of $H$ given by Lemma 5.5(i) and let $\tau:=-\ln \xi$. Then, by Lemma 5.5, $H\left(e^{-t}\right)>0$ for $0<t<\tau$, and thus $\inf _{t \leq \tau} H\left(e^{-t}\right)=0$. Consequently, (5.6) implies

$$
n^{-1} \inf _{t \leq \tau} \widetilde{A}(t)=\inf _{t \leq \tau} n^{-1} \widetilde{A}(t)-\inf _{t \leq \tau} H\left(e^{-t}\right) \stackrel{\mathrm{p}}{\longrightarrow} 0 .
$$

Further, by Condition 2.1(iii), $d_{\max }=O\left(n^{1 / 2}\right)$, and thus $n^{-1} d_{\max } \rightarrow 0$. Consequently, (5.7) and (5.8) yield

$$
\sup _{t \leq \tau} n^{-1}|A(t)-\widetilde{A}(t)|=\sup _{t \leq \tau} n^{-1}|\widetilde{S}(t)-S(t)| \stackrel{\mathrm{p}}{\longrightarrow} 0
$$


and thus, by (5.6),

$$
\sup _{t \leq \tau}\left|n^{-1} A(t)-H\left(e^{-t}\right)\right| \stackrel{\mathrm{p}}{\longrightarrow} 0 .
$$

Let $0<\varepsilon<\tau / 2$. Since $H\left(e^{-t}\right)>0$ on the compact interval $[\varepsilon, \tau-\varepsilon]$, (5.10) implies that whp $A(t)$ remains positive on $[\varepsilon, \tau-\varepsilon]$, and thus no new component is started during this interval.

On the other hand, again by Lemma 5.5(i), $H\left(e^{-\tau-\varepsilon}\right)<0$ and (5.6) implies $n^{-1} \widetilde{A}(\tau+\varepsilon) \stackrel{\mathrm{p}}{\longrightarrow} H\left(e^{-\tau-\varepsilon}\right)$, while $A(\tau+\varepsilon) \geq 0$. Thus, with $\delta:=$ $\left|H\left(e^{-\tau-\varepsilon}\right)\right| / 2>0$, whp

$$
\widetilde{S}(\tau+\varepsilon)-S(\tau+\varepsilon)=A(\tau+\varepsilon)-\widetilde{A}(\tau+\varepsilon) \geq-\widetilde{A}(\tau+\varepsilon)>n \delta,
$$

while $(5.9)$ yields $\widetilde{S}(\tau)-S(\tau)<n \delta$ whp. Consequently, whp $\widetilde{S}(\tau+\varepsilon)-$ $S(\tau+\varepsilon)>\widetilde{S}(\tau)-S(\tau)$, so $C 1$ is performed between $\tau$ and $\tau+\varepsilon$.

Let $T_{1}$ be the last time C1 was performed before $\tau / 2$ and let $T_{2}$ be the next time it is performed. We have shown that for any $\varepsilon>0$, whp $0 \leq T_{1} \leq \varepsilon$ and $\tau-\varepsilon \leq T_{2} \leq \tau+\varepsilon$; in other words, $T_{1} \stackrel{\mathrm{p}}{\longrightarrow} 0$ and $T_{2} \stackrel{\mathrm{p}}{\longrightarrow} \tau$.

We state the next step as a lemma that we will reuse.

Lemma 5.6. Let $T_{1}^{*}$ and $T_{2}^{*}$ be two (random) times when $\mathrm{C} 1$ are performed, with $T_{1}^{*} \leq T_{2}^{*}$, and assume that $T_{1}^{*} \stackrel{\mathrm{p}}{\longrightarrow} t_{1}$ and $T_{2}^{*} \stackrel{\mathrm{p}}{\longrightarrow} t_{2}$ where $0 \leq t_{1} \leq$ $t_{2} \leq \tau$. If $C^{*}$ is the union of all components explored between $T_{1}^{*}$ and $T_{2}^{*}$, then

$$
\begin{aligned}
& v_{k}\left(C^{*}\right) / n \stackrel{\mathrm{p}}{\longrightarrow} p_{k}\left(e^{-k t_{1}}-e^{-k t_{2}}\right), \quad k \geq 0, \\
& v\left(C^{*}\right) / n \stackrel{\mathrm{p}}{\longrightarrow} g\left(e^{-t_{1}}\right)-g\left(e^{-t_{2}}\right), \\
& e\left(C^{*}\right) / n \stackrel{\mathrm{p}}{\longrightarrow} \frac{1}{2} h\left(e^{-t_{1}}\right)-\frac{1}{2} h\left(e^{-t_{2}}\right) .
\end{aligned}
$$

In particular, if $t_{1}=t_{2}$, then $v\left(C^{*}\right) / n \stackrel{\mathrm{p}}{\longrightarrow} 0$ and $e\left(C^{*}\right) / n \stackrel{\mathrm{p}}{\longrightarrow} 0$.

Proof. $C^{*}$ contains all vertices awakened in the interval $\left[T_{1}^{*}, T_{2}^{*}\right)$ and no others, and thus

$$
v_{k}\left(C^{*}\right)=V_{k}\left(T_{1}^{*}-\right)-V_{k}\left(T_{2}^{*}-\right), \quad k \geq 1 .
$$

Since $T_{2}^{*} \stackrel{\mathrm{p}}{\longrightarrow} t_{2} \leq \tau$ and $H$ is continuous, $\inf _{t \leq T_{2}^{*}} H(t) \stackrel{\mathrm{p}}{\longrightarrow} \inf _{t \leq t_{2}} H(t)=$ 0 , and (5.6) and (5.7) imply, in analogy with (5.8) and (5.9), $n^{-1} \inf _{t \leq T_{2}^{*}} \widetilde{A}(t) \stackrel{\mathrm{p}}{\longrightarrow}$ 0 and

$$
\sup _{t \leq T_{2}^{*}} n^{-1}|\widetilde{S}(t)-S(t)| \stackrel{\mathrm{p}}{\longrightarrow} 0 .
$$

Since $\widetilde{V}_{j}(t) \geq V_{j}(t)$ for every $j$ and $t \geq 0$,

$$
\widetilde{V}_{k}(t)-V_{k}(t) \leq k^{-1} \sum_{j=1}^{\infty} j\left(\widetilde{V}_{j}(t)-V_{j}(t)\right)=k^{-1}(\widetilde{S}(t)-S(t)), \quad k \geq 1 .
$$


Hence (5.16) implies, for every $k \geq 1, \sup _{t \leq T_{2}^{*}}\left|\widetilde{V}_{k}(t)-V_{k}(t)\right|=o_{\mathrm{p}}(n)$. This is further trivially true for $k=0$ too. Consequently, using Lemma 5.2, for $j=1,2$,

$$
V_{k}\left(T_{j}^{*}-\right)=\widetilde{V}_{k}\left(T_{j}^{*}-\right)+o_{\mathrm{p}}(n)=n p_{k} e^{-k T_{j}^{*}}+o_{\mathrm{p}}(n)=n p_{k} e^{-k t_{j}}+o_{\mathrm{p}}(n),
$$

and (5.12) follows by (5.15). Similarly, using $\sum_{k=0}^{\infty}\left(\widetilde{V}_{k}(t)-V_{k}(t)\right) \leq \widetilde{S}(t)-$ $S(t)$,

$$
\begin{aligned}
v\left(C^{*}\right) & =\sum_{k=1}^{\infty}\left(V_{k}\left(T_{1}^{*}-\right)-V_{k}\left(T_{2}^{*}-\right)\right)=\sum_{k=1}^{\infty}\left(\widetilde{V}_{k}\left(T_{1}^{*}-\right)-\widetilde{V}_{k}\left(T_{2}^{*}-\right)\right)+o_{\mathrm{p}}(n) \\
& =n g\left(e^{-T_{1}^{*}}\right)-n g\left(e^{-T_{2}^{*}}\right)+o_{\mathrm{p}}(n)
\end{aligned}
$$

and

$$
\begin{aligned}
2 e\left(C^{*}\right) & =\sum_{k=1}^{\infty} k\left(V_{k}\left(T_{1}^{*}-\right)-V_{k}\left(T_{2}^{*}-\right)\right)=\sum_{k=1}^{\infty} k\left(\widetilde{V}_{k}\left(T_{1}^{*}-\right)-\widetilde{V}_{k}\left(T_{2}^{*}-\right)\right)+o_{\mathrm{p}}(n) \\
& =n h\left(e^{-T_{1}^{*}}\right)-n h\left(e^{-T_{2}^{*}}\right)+o_{\mathrm{p}}(n),
\end{aligned}
$$

and (5.13) and (5.14) follow.

Let $\mathcal{C}^{\prime}$ be the component created at $T_{1}$ and explored until $T_{2}$. By Lemma 5.6, with $t_{1}=0$ and $t_{2}=\tau$,

$$
\begin{aligned}
& v_{k}\left(\mathcal{C}^{\prime}\right) / n \stackrel{\mathrm{p}}{\longrightarrow} p_{k}\left(1-e^{-k \tau}\right), \\
& v\left(\mathcal{C}^{\prime}\right) / n \stackrel{\mathrm{p}}{\longrightarrow} g(1)-g\left(e^{-\tau}\right)=1-g(\xi), \\
& e\left(\mathcal{C}^{\prime}\right) / n \stackrel{\mathrm{p}}{\longrightarrow} \frac{1}{2}\left(h(1)-h\left(e^{-\tau}\right)\right)=\frac{1}{2}(h(1)-h(\xi))=\frac{\lambda}{2}\left(1-\xi^{2}\right),
\end{aligned}
$$

using (2.7) and $H(1)=H(\xi)=0$.

We have found one large component $\mathcal{C}^{\prime}$ with the claimed numbers of vertices and edges. It remains to show that there is whp no other large component. Therefore, let $T_{3}$ be the first time after $T_{2}$ that $C 1$ is performed. Since $\widetilde{S}(t)-S(t)$ increases by at most $d_{\max }=o_{\mathrm{p}}(n)$ each time $\mathrm{C} 1$ is performed, we obtain from (5.16) that

$$
\sup _{t \leq T_{3}}(\widetilde{S}(t)-S(t)) \leq \sup _{t \leq T_{2}}(\widetilde{S}(t)-S(t))+d_{\max }=o_{\mathrm{p}}(n) .
$$

Comparing this to (5.11) we see that for every $\varepsilon>0$, whp $\tau+\varepsilon>T_{3}$. Since also $T_{3}>T_{2} \stackrel{\mathrm{p}}{\longrightarrow} \tau$, it follows that $T_{3} \stackrel{\mathrm{p}}{\longrightarrow} \tau$. If $\mathcal{C}^{\prime \prime}$ is the component created between $T_{2}$ and $T_{3}$, then Lemma 5.6 applied to $T_{2}$ and $T_{3}$ yields $v\left(\mathcal{C}^{\prime \prime}\right) / n \stackrel{\mathrm{p}}{\longrightarrow} 0$ and $e\left(\mathcal{C}^{\prime \prime}\right) / n \stackrel{\mathrm{p}}{\longrightarrow} 0$.

Next, let $\eta>0$. Applying Lemma 5.6 to $T_{0}:=0$ and $T_{1}$, we see that the total number of vertices and edges in all components found before $\mathcal{C}^{\prime}$, i.e., before $T_{1}$, is $o_{\mathrm{p}}(n)$, because $T_{1} \stackrel{\mathrm{p}}{\longrightarrow} 0$. Hence, recalling $m=\Theta(n)$ by $(2.4)$,

$$
\mathbb{P}\left(\text { a component } \mathcal{C} \text { with } e(\mathcal{C}) \geq \eta m \text { is found before } \mathcal{C}^{\prime}\right) \rightarrow 0 \text {. }
$$


On the other hand, conditioning on the final graph $G^{*}\left(n,\left(d_{i}\right)_{1}^{n}\right)$ that is constructed by the algorithm, if there exists a component $\mathcal{C} \neq \mathcal{C}^{\prime}$ in $G^{*}\left(n,\left(d_{i}\right)_{1}^{n}\right)$ with at least $\eta m$ edges that has not been found before $\mathcal{C}^{\prime}$, then with probability at least $\eta$, the vertex chosen at random by $\mathrm{C} 1$ at $T_{2}$ starting the component $\mathcal{C}^{\prime \prime}$ belongs to $\mathcal{C}$, and thus $\mathcal{C}=\mathcal{C}^{\prime \prime}$. Consequently,

$\mathbb{P}\left(\right.$ a component $\mathcal{C}$ with $e(\mathcal{C}) \geq \eta m$ is found after $\left.\mathcal{C}^{\prime}\right)$

$$
\leq \eta^{-1} \mathbb{P}\left(e\left(\mathcal{C}^{\prime \prime}\right) \geq \eta m\right) \rightarrow 0 .
$$

Combining (5.22) and (5.23), we see that whp there is no component except $\mathcal{C}^{\prime}$ with at least $\eta m$ edges. Taking $\eta$ small, this and (5.21) show that whp $\mathcal{C}^{\prime}=\mathcal{C}_{1}$, the largest component, and further $e\left(\mathcal{C}_{2}\right)<\eta m$. Consequently, the results for $\mathcal{C}_{1}$ follow from (5.19)-(5.21). We have further shown $e\left(\mathcal{C}_{2}\right) / m \stackrel{\mathrm{p}}{\longrightarrow}$ 0 , which implies $e\left(\mathcal{C}_{2}\right) / n \stackrel{\mathrm{p}}{\longrightarrow} 0$ and $v\left(\mathcal{C}_{2}\right) / n \stackrel{\mathrm{p}}{\longrightarrow} 0$ because $m=O(n)$ and $v\left(\mathcal{C}_{2}\right) \leq e\left(\mathcal{C}_{2}\right)+1$.

Proof of Theorem 2.3(ii). This is very similar to the last step in the proof for (i). Let $T_{1}=0$ and let $T_{2}$ be the next time $\mathrm{C} 1$ is performed. Then

$$
\sup _{t \leq T_{2}}|A(t)-\widetilde{A}(t)|=\sup _{t \leq T_{2}}|\widetilde{S}(t)-S(t)| \leq 2 d_{\max }=o(n) .
$$

For every $\varepsilon>0$, we have by (5.6) and Lemma $5.5(\mathrm{ii}) n^{-1} \widetilde{A}(\varepsilon) \stackrel{\mathrm{p}}{\longrightarrow} H\left(e^{-\varepsilon}\right)<$ 0 , while $A(\varepsilon) \geq 0$, and it follows from (5.24) that whp $T_{2}<\varepsilon$. Hence, $T_{2} \stackrel{\mathrm{p}}{\longrightarrow} 0$. We apply Lemma 5.6 (which holds in this case too, with $\tau=0$ ) and find that if $\widetilde{\mathcal{C}}$ is the first component, then $e(\widetilde{\mathcal{C}}) / n \stackrel{\mathrm{p}}{\longrightarrow} 0$.

Let $\varepsilon>0$. If $e\left(\mathcal{C}_{1}\right) \geq \varepsilon m$, then the probability that the first half-edge chosen by $\mathrm{C} 1$ belongs to $\mathcal{C}_{1}$, and thus $\widetilde{\mathcal{C}}=\mathcal{C}_{1}$, is $2 e\left(\mathcal{C}_{1}\right) /(2 m) \geq \varepsilon$, and hence, using $m=\Theta(n)$ by $(2.4)$,

$$
\mathbb{P}\left(e\left(\mathcal{C}_{1}\right) \geq \varepsilon m\right) \leq \varepsilon^{-1} \mathbb{P}(e(\widetilde{\mathcal{C}}) \geq \varepsilon m) \rightarrow 0 .
$$

The results follows since $m=O(n)$ and $v\left(\mathcal{C}_{1}\right) \leq e\left(\mathcal{C}_{1}\right)+1$.

\section{Proof of Theorem 2.4 For $G^{*}\left(n,\left(d_{i}\right)_{1}^{n}\right)$}

We assume in this section that the assumptions of Theorem 2.4 hold. Note first that $\beta:=\mathbb{E} D(D-1)(D-2) \geq 0$ with strict inequality unless $\mathbb{P}(D \leq 2)=1$, but in the latter case $\alpha=\mathbb{E} D(D-2)=-p_{1}<0$, which is ruled out by the assumptions.

Define, in analogy with (2.5)-(2.7),

$$
\begin{aligned}
& g_{n}(x):=\mathbb{E} x^{D_{n}}=\sum_{k=0}^{\infty} \frac{n_{k}}{n} x^{k}, \\
& h_{n}(x):=x g_{n}^{\prime}(x)=\sum_{k=1}^{\infty} k \frac{n_{k}}{n} x^{k},
\end{aligned}
$$




$$
H_{n}(x):=\frac{2 m(n)}{n} x^{2}-h_{n}(x)=\sum_{k=1}^{\infty} k \frac{n_{k}}{n}\left(x^{2}-x^{k}\right)=\mathbb{E} D_{n}\left(x^{2}-x^{D_{n}}\right) .
$$

We begin with a general estimate for death processes and use it to prove estimates improving Lemmas 5.1 and 5.2.

Lemma 6.1. Let $\gamma>0$ and $d>0$ be fixed. Let $N^{(x)}(t)$ be a Markov process such that $N^{(x)}(0)=x$ a.s. and transitions are made according to the following rule: whenever in state $y>0$, the process jumps to $y-d$ with intensity $\gamma y$; in other words, the waiting time until the next event is $\operatorname{Exp}(1 / \gamma y)$ and each jump is of size $d$ downwards. Then, for every $t_{0} \geq 0$,

$$
\mathbb{E} \sup _{t \leq t_{0}}\left|N^{(x)}(t)-e^{-\gamma d t} x\right|^{2} \leq 8 d\left(e^{\gamma d t_{0}}-1\right) x+8 d^{2} .
$$

If $x / d$ is an integer, we also have the better estimate

$$
\mathbb{E} \sup _{t \leq t_{0}}\left|N^{(x)}(t)-e^{-\gamma d t} x\right|^{2} \leq 4 d\left(e^{\gamma d t_{0}}-1\right) x .
$$

Proof. First assume that $d=1$ and that $x$ is an integer. In this case, the process is a standard pure death process taking the values $x, x-1, \ldots, 0$, describing the number of particles alive when the particles die independently with rate $\gamma$. As is well-known, and easily seen by regarding $N^{(x)}(t)$ as the sum of $x$ independent copies of the process $N^{(1)}(t)$, the process $e^{\gamma t} N^{(x)}(t)$, $t \geq 0$, is a martingale. Furthermore, for every $t \geq 0, N^{(x)}(t) \sim \operatorname{Bi}\left(x, e^{-\gamma t}\right)$. Hence, by Doob's inequality,

$$
\begin{aligned}
\mathbb{E} \sup _{t \leq t_{0}}\left|N^{(x)}(t)-x e^{-\gamma t}\right|^{2} & \leq \mathbb{E} \sup _{t \leq t_{0}}\left|e^{\gamma t} N^{(x)}(t)-x\right|^{2} \leq 4 \mathbb{E}\left|e^{\gamma t_{0}} N^{(x)}\left(t_{0}\right)-x\right|^{2} \\
& =4 e^{2 \gamma t_{0}} \operatorname{Var} N^{(x)}\left(t_{0}\right)=4\left(e^{\gamma t_{0}}-1\right) x
\end{aligned}
$$

Next, still assume $d=1$ but let $x \geq 0$ be arbitrary. We can couple the two processes $N^{(x)}(t)$ and $N^{(\lfloor x\rfloor)}(t)$ with different initial values such that whenever the smaller one jumps (by -1 ), so does the other. This coupling keeps $\left|N^{(x)}(t)-N^{(\lfloor x\rfloor)}(t)\right|<1$ for all $t \geq 0$, and thus,

$$
\sup _{t \leq t_{0}}\left|N^{(x)}(t)-x e^{-\gamma t}\right| \leq \sup _{t \leq t_{0}}\left|N^{(\lfloor x\rfloor)}(t)-\lfloor x\rfloor e^{-\gamma t}\right|+2
$$

and hence by $(6.3)$

$$
\mathbb{E} \sup _{t \leq t_{0}}\left|N^{(x)}(t)-x e^{-\gamma t}\right|^{2} \leq 8\left(e^{\gamma t_{0}}-1\right) x+8 .
$$

Finally, for a general $d>0$ we observe that $N^{(x)}(t) / d$ is a process of the same type with the parameters $(\gamma, d, x)$ replaced by $(\gamma d, 1, x / d)$, and the general result follows from (6.4) and (6.3).

Lemma 6.2. For every fixed $t_{0}>0$, as $n \rightarrow \infty$,

$$
\sup _{t \leq \alpha_{n} t_{0}}\left|L(t)-2 m(n) e^{-2 t}\right|=O_{\mathrm{p}}\left(n^{1 / 2} \alpha_{n}^{1 / 2}+1\right) .
$$


Proof. $L(t)$ is a death process as in Lemma 6.1 , with $\gamma=1, d=2$ and $x=L(0)=2 m(n)-1$. Hence, by Lemma 6.1 applied to $\alpha_{n} t_{0}$, observing that $\alpha_{n} t_{0}=O\left(\alpha_{n}\right)=O(1)$ and $m(n)=O(n)$,

$$
\mathbb{E} \sup _{t \leq \alpha_{n} t_{0}}\left|L(t)-2 m(n) e^{-2 t}\right|^{2}=O\left(\left(e^{2 \alpha_{n} t_{0}}-1\right) m(n)+1\right)=O\left(\alpha_{n} n+1\right) .
$$

Lemma 6.3. For every fixed $t_{0} \geq 0$

$$
\sup _{t \leq \alpha_{n} t_{0}}\left|\widetilde{V}_{k}(t)-n_{k} e^{-k t}\right|=O_{\mathrm{p}}\left(n^{1 / 2} \alpha_{n}^{1 / 2}\right)
$$

for every $k \geq 0$ and

$$
\begin{gathered}
\sup _{t \leq \alpha_{n} t_{0}}\left|\sum_{k=0}^{\infty} \widetilde{V}_{k}(t)-n g_{n}\left(e^{-t}\right)\right|=O_{\mathrm{p}}\left(n^{1 / 2} \alpha_{n}^{1 / 2}+n \alpha_{n}^{3}\right), \\
\sup _{t \leq \alpha_{n} t_{0}}\left|\widetilde{S}(t)-n h_{n}\left(e^{-t}\right)\right|=O_{\mathrm{p}}\left(n^{1 / 2} \alpha_{n}^{1 / 2}+n \alpha_{n}^{3}\right) .
\end{gathered}
$$

Proof. $\widetilde{V}_{k}(t)$ is a death process as in Lemma 6.1 with $\gamma=k, d=1$ and $x=n_{k}$. Consequently, by (6.2) (for $k \geq 1$; the case $k=0$ is trivial),

$$
\mathbb{E} \sup _{t \leq \alpha_{n} t_{0}}\left|\widetilde{V}_{k}(t)-n_{k} e^{-k t}\right|^{2} \leq 4\left(e^{k \alpha_{n} t_{0}}-1\right) n_{k} \leq 4 k \alpha_{n} t_{0} e^{k \alpha_{n} t_{0}} n_{k}
$$

The estimate for fixed $k$ follows immediately.

To treat $\widetilde{S}(t)=\sum_{k=1}^{\infty} k \widetilde{V}_{k}(t)$, we use (6.5) for $k \leq \alpha_{n}^{-1}$ and obtain

$$
\mathbb{E} \sup _{t \leq \alpha_{n} t_{0}}\left|\widetilde{V}_{k}(t)-n_{k} e^{-k t}\right| \leq\left(4 t_{0} e^{t_{0}} k \alpha_{n} n_{k}\right)^{1 / 2}, \quad k \leq \alpha_{n}^{-1} .
$$

For $k>\alpha_{n}^{-1}$ we use the trivial estimate $\sup _{t}\left|\widetilde{V}_{k}(t)-n_{k} e^{-k t}\right| \leq n_{k}$. Summing over $k$ and using the Cauchy-Schwarz inequality and (2.9) we find, for some $C$ depending on $t_{0}$,

$$
\begin{aligned}
\mathbb{E} \sup _{t \leq \alpha_{n} t_{0}} \mid \widetilde{S}(t) & -\sum_{k=1}^{\infty} k n_{k} e^{-k t}\left|\leq \mathbb{E} \sum_{k=1}^{\infty} k \sup _{t \leq \alpha_{n} t_{0}}\right| \widetilde{V}_{k}(t)-n_{k} e^{-k t} \mid \\
\leq & C \sum_{k \leq \alpha_{n}^{-1}} k\left(k \alpha_{n} n_{k}\right)^{1 / 2}+\sum_{k>\alpha_{n}^{-1}} k n_{k} \\
\leq & C \alpha_{n}^{1 / 2}\left(\sum_{k=1}^{\infty} k^{4+\eta} n_{k}\right)^{1 / 2}\left(\sum_{k=1}^{\infty} k^{-1-\eta}\right)^{1 / 2}+\alpha_{n}^{3} \sum_{k>\alpha_{n}^{-1}} k^{4} n_{k} \\
& =O\left(n^{1 / 2} \alpha_{n}^{1 / 2}+n \alpha_{n}^{3}\right) .
\end{aligned}
$$

The estimate for $\sum_{k=0}^{\infty} \widetilde{V}_{k}(t)$ is proved the same way. 
Lemmas 6.2 and 6.3 imply, cf. (5.5) and (5.6), for every $t_{0}>0$,

$$
\begin{aligned}
\alpha_{n}^{-2} \sup _{t \leq t_{0}} \mid n^{-1} \widetilde{A}\left(\alpha_{n} t\right) & -H_{n}\left(e^{-\alpha_{n} t}\right) \mid \\
= & \alpha_{n}^{-2} n^{-1} \sup _{t \leq \alpha_{n} t_{0}}\left|L(t)-\widetilde{S}(t)-n H_{n}\left(e^{-t}\right)\right| \\
= & O_{\mathrm{p}}\left(n^{-1 / 2} \alpha_{n}^{-3 / 2}+n^{-1} \alpha_{n}^{-2}+\alpha_{n}\right)=o_{\mathrm{p}}(1),
\end{aligned}
$$

recalling $\alpha_{n} \rightarrow 0$ by Remark 2.5 and $n \alpha_{n}^{3} \rightarrow \infty$.

Let

$$
\check{H}_{n}(t):=H_{n}\left(e^{-t}\right)=\mathbb{E} D_{n}\left(e^{-2 t}-e^{-t D_{n}}\right) .
$$

Then $\check{H}_{n}(0)=0, \check{H}_{n}^{\prime}(0)=\mathbb{E} D_{n}\left(D_{n}-2\right)=\alpha_{n}, \check{H}_{n}^{\prime \prime}(0)=\mathbb{E} D_{n}\left(4-D_{n}^{2}\right)=$ $-\mathbb{E} D_{n}\left(D_{n}+2\right)\left(D_{n}-2\right)$, and for all $t \geq 0$,

$$
\left|\check{H}_{n}^{\prime \prime \prime}(t)\right|=\left|\mathbb{E}\left(D_{n}\left(8 e^{-2 t}-D_{n}^{3} e^{-t D_{n}}\right)\right)\right| \leq \mathbb{E}\left(8 D_{n}+D_{n}^{4}\right)=O(1) .
$$

Moreover, using Remark 2.5,

$$
\begin{aligned}
\check{H}_{n}^{\prime \prime}(0) & =-\mathbb{E} D_{n}\left(D_{n}+2\right)\left(D_{n}-2\right) \\
& \rightarrow-\mathbb{E} D(D+2)(D-2) \\
& =-\mathbb{E} D(D-1)(D-2)-3 \mathbb{E} D(D-2)=-\beta .
\end{aligned}
$$

Hence a Taylor expansion yields, for $t \geq 0$,

$$
\check{H}_{n}\left(\alpha_{n} t\right)=\alpha_{n}^{2} t+\frac{1}{2} \check{H}_{n}^{\prime \prime}(0)\left(\alpha_{n} t\right)^{2}+O\left(\left(\alpha_{n} t\right)^{3}\right)=\alpha_{n}^{2}\left(t-\frac{1}{2} \beta t^{2}+o\left(t^{2}+t^{3}\right)\right) .
$$

Consequently, (6.6) yields, for every fixed $t_{0}>0$,

$$
\sup _{t \leq t_{0}}\left|\alpha_{n}^{-2} n^{-1} \widetilde{A}\left(\alpha_{n} t\right)-\left(t-\frac{1}{2} \beta t^{2}\right)\right|=o_{\mathrm{p}}(1) .
$$

We now proceed as in the proof of Theorem 2.3, using $\widetilde{H}(t):=t-\frac{1}{2} \beta t^{2}$ instead of $H\left(e^{-t}\right)$. We note that $\widetilde{H}(t)>0$ for $0<t<2 / \beta$ and $\widetilde{H}(t)<0$ for $t>2 / \beta$; thus we now define $\tau=2 / \beta$. We obtain from (6.7), for any random $T \stackrel{\mathrm{p}}{\longrightarrow} \tau$,

$$
\alpha_{n}^{-2} n^{-1} \inf _{t \leq T} \widetilde{A}\left(\alpha_{n} t\right) \stackrel{\mathrm{p}}{\longrightarrow} 0
$$

and, using (5.7), since by $(2.9) d_{\max }=o\left(n^{1 / 3}\right)=o\left(n \alpha_{n}^{2}\right)$,

$$
\alpha_{n}^{-2} n^{-1} \sup _{t \leq T}\left|A\left(\alpha_{n} t\right)-\widetilde{A}\left(\alpha_{n} t\right)\right|=\alpha_{n}^{-2} n^{-1} \sup _{t \leq T}\left|\widetilde{S}\left(\alpha_{n} t\right)-S\left(\alpha_{n} t\right)\right| \stackrel{\mathrm{p}}{\longrightarrow} 0
$$

and thus, by (6.7) again,

$$
\sup _{t \leq T}\left|\alpha_{n}^{-2} n^{-1} A\left(\alpha_{n} t\right)-\widetilde{H}(t)\right| \stackrel{\mathrm{p}}{\longrightarrow} 0 .
$$

Taking $T=\tau$, it follows as in Section 5 that whp there is a component $\mathcal{C}^{\prime}$ explored between two random times $T_{1}$ and $T_{2}$ with $T_{1} / \alpha_{n} \stackrel{\mathrm{p}}{\longrightarrow} 0$ and $T_{2} / \alpha_{n} \stackrel{\mathrm{p}}{\longrightarrow} \tau=2 / \beta$. We have the following analogue of Lemma 5.6. 
Lemma 6.4. Let $T_{1}$ and $T_{2}$ be two (random) times when $\mathrm{C} 1$ are performed, with $T_{1} \leq T_{2}$, and assume that $T_{1} / \alpha_{n} \stackrel{\mathrm{p}}{\longrightarrow} t_{1}$ and $T_{2} / \alpha_{n} \stackrel{\mathrm{p}}{\longrightarrow} t_{2}$ where $0 \leq t_{1} \leq t_{2} \leq \tau=2 / \beta$. If $\widetilde{\mathcal{C}}$ is the union of all components explored between $T_{1}$ and $T_{2}$, then

$$
\begin{aligned}
v_{k}(\widetilde{\mathcal{C}}) & =n \alpha_{n} k p_{k}\left(t_{2}-t_{1}\right)+o_{\mathrm{p}}\left(n \alpha_{n}\right), \quad k \geq 0, \\
v(\widetilde{\mathcal{C}}) & =n \alpha_{n} \lambda\left(t_{2}-t_{1}\right)+o_{\mathrm{p}}\left(n \alpha_{n}\right), \\
e(\widetilde{\mathcal{C}}) & =n \alpha_{n} \lambda\left(t_{2}-t_{1}\right)+o_{\mathrm{p}}\left(n \alpha_{n}\right) .
\end{aligned}
$$

In particular, if $t_{1}=t_{2}$, then $v(\widetilde{\mathcal{C}})=o_{\mathrm{p}}\left(n \alpha_{n}\right)$ and $e(\widetilde{\mathcal{C}})=o_{\mathrm{p}}\left(n \alpha_{n}\right)$.

Proof. Taking, for $j=1,2, T=T_{j} / \alpha_{n}+\tau-t_{j}$ in (6.8), we see that $\widetilde{S}\left(T_{j}\right)-$ $S\left(T_{j}\right)=o_{\mathrm{p}}\left(n \alpha_{n}^{2}\right)$. Since further, for every $k \geq 1,0 \leq \widetilde{V}_{k}\left(T_{j}\right)-V_{k}\left(T_{j}\right) \leq$ $\widetilde{S}\left(T_{j}\right)-S\left(T_{j}\right)$, we have

$$
\widetilde{V}\left(T_{j}\right)-V\left(T_{j}\right)=o_{\mathrm{p}}\left(n \alpha_{n}^{2}\right) .
$$

$\widetilde{\mathcal{C}}$ consists of the vertices awakened in the interval $\left[T_{1}, T_{2}\right)$, and thus, using (6.9) and Lemma 6.3,

$$
\begin{aligned}
v_{k}(\widetilde{\mathcal{C}}) & =V_{k}\left(T_{1}-\right)-V_{k}\left(T_{2}-\right)=\widetilde{V}_{k}\left(T_{1}-\right)-\widetilde{V}_{k}\left(T_{2}-\right)+o_{\mathrm{p}}\left(n \alpha_{n}^{2}\right) \\
& =n_{k}\left(e^{-k T_{1}}-e^{-k T_{2}}\right)+o_{\mathrm{p}}\left(n \alpha_{n}^{2}\right) \\
& =n_{k}\left(k T_{2}-k T_{1}+O_{\mathrm{p}}\left(\alpha_{n}^{2}\right)\right)+o_{\mathrm{p}}\left(n \alpha_{n}^{2}\right) \\
& =n_{k} k \alpha_{n}\left(t_{2}-t_{1}\right)+o_{\mathrm{p}}\left(n \alpha_{n}\right) \\
& =k p_{k} n \alpha_{n}\left(t_{2}-t_{1}\right)+o_{\mathrm{p}}\left(n \alpha_{n}\right) .
\end{aligned}
$$

Further, since $g_{n}^{\prime}(1)=\mathbb{E} D_{n} \rightarrow \mathbb{E} D=\lambda$ and $g_{n}^{\prime \prime}(x)=O(1)$ for $0<x<1$, a Taylor expansion yields, for $j=1,2$,

$$
g_{n}\left(e^{-T_{j}}\right)=g_{n}(1)-g_{n}^{\prime}(1) T_{j}+O\left(T_{j}^{2}\right)=1-\lambda \alpha_{n} t_{j}+o_{\mathrm{p}}\left(\alpha_{n}\right) .
$$

Similarly, since $h_{n}^{\prime}(1)=\mathbb{E} D_{n}^{2}=\alpha_{n}+2 \mathbb{E} D_{n} \rightarrow 2 \lambda$, and $h_{n}^{\prime \prime}(x)=O(1)$ for $0<x<1$,

$$
h_{n}\left(e^{-T_{j}}\right)=h_{n}(1)-h_{n}^{\prime}(1) T_{j}+O\left(T_{j}^{2}\right)=\mathbb{E} D_{n}-2 \lambda \alpha_{n} t_{j}+o_{\mathrm{p}}\left(\alpha_{n}\right) .
$$

It now follows from (6.8) and Lemma 6.3 that

$$
\begin{aligned}
v(\widetilde{\mathcal{C}}) & =n g_{n}\left(e^{-T_{1}}\right)-n g_{n}\left(e^{-T_{2}}\right)+o_{\mathrm{p}}\left(n \alpha_{n}^{2}\right)=n \alpha_{n} \lambda\left(t_{2}-t_{1}\right)+o_{\mathrm{p}}\left(n \alpha_{n}\right), \\
2 e(\widetilde{\mathcal{C}}) & =n h_{n}\left(e^{-T_{1}}\right)-n h_{n}\left(e^{-T_{2}}\right)+o_{\mathrm{p}}\left(n \alpha_{n}^{2}\right)=2 n \alpha_{n} \lambda\left(t_{2}-t_{1}\right)+o_{\mathrm{p}}\left(n \alpha_{n}\right) .
\end{aligned}
$$

In particular, for the component $\mathcal{C}^{\prime}$ found above, with $t_{1}=0$ and $t_{2}=\tau$,

$$
\begin{aligned}
v_{k}\left(\mathcal{C}^{\prime}\right) & =k p_{k} \tau n \alpha_{n}+o_{\mathrm{p}}\left(n \alpha_{n}\right), \\
v\left(\mathcal{C}^{\prime}\right) & =\lambda \tau n \alpha_{n}+o_{\mathrm{p}}\left(n \alpha_{n}\right), \\
e\left(\mathcal{C}^{\prime}\right) & =\lambda \tau n \alpha_{n}+o_{\mathrm{p}}\left(n \alpha_{n}\right) .
\end{aligned}
$$


Since $\tau=2 / \beta$, these are the estimates we claim for $\mathcal{C}_{1}$, and it remains only to show that whp all other components are much smaller than $\mathcal{C}^{\prime}$.

Fix $\varepsilon>0$ with $\varepsilon<\tau$, and say that a component of $G^{*}\left(n,\left(d_{i}\right)_{1}^{n}\right)$ is large if it has at least $\varepsilon m \alpha_{n}$ edges $\left(2 \varepsilon m \alpha_{n}\right.$ half-edges). Since, by (6.12) and (2.4), $e\left(\mathcal{C}^{\prime}\right) /\left(m \alpha_{n}\right) \stackrel{\mathrm{p}}{\longrightarrow} 2 \tau$, whp $\mathcal{C}^{\prime}$ is large, and further $(2 \tau-\varepsilon) m \alpha_{n}<e\left(\mathcal{C}^{\prime}\right)<$ $(2 \tau+\varepsilon) m \alpha_{n}$. Let $\mathcal{E}_{\varepsilon}$ be the event that $e\left(\mathcal{C}^{\prime}\right)<(2 \tau+\varepsilon) m \alpha_{n}$ and that the total number of edges in large components is at least $(2 \tau+2 \varepsilon) m \alpha_{n}$.

It follows by Lemma 6.4 applied to $T_{0}=0$ and $T_{1}$ that the total number of vertices or edges in components found before $\mathcal{C}^{\prime}$ is $o_{\mathrm{p}}\left(n \alpha_{n}\right)$. Thus there exists a sequence $\alpha_{n}^{\prime}$ of constants such that $\alpha_{n}^{\prime}=o\left(\alpha_{n}\right)$ and whp at most $n \alpha_{n}^{\prime}$ vertices are found before $T_{1}$, when the first large component is found.

Let us now condition on the final graph obtained through our componentfinding algorithm. Given $G^{*}\left(n,\left(d_{i}\right)_{1}^{n}\right)$, the components appear in our process in the size-biased order (with respect to the number of edges) obtained by picking half-edges uniformly at random (with replacement, for simplicity) and taking the corresponding components, ignoring every component that already has been taken. We have seen that whp this finds component containing at most $n \alpha_{n}^{\prime}$ vertices before a half-edge in a large component is picked. Therefore, starting again at $T_{2}$, whp we find at most $n \alpha_{n}^{\prime}$ vertices in new components before a half-edge is chosen in some large component; this half-edge may belong to $\mathcal{C}^{\prime}$, but if $\mathcal{E}_{\varepsilon}$ holds, then with probability at least $\varepsilon_{1}:=1-(2 \tau+\varepsilon) /(2 \tau+2 \varepsilon)$ it does not, and therefore it belongs to a new large component. Consequently, with probability at least $\varepsilon_{1} \mathbb{P}\left(\mathcal{E}_{\varepsilon}\right)+o(1)$, the algorithm in Section 4 finds a second large component at a time $T_{3}$, and less than $n \alpha_{n}^{\prime}$ vertices between $T_{2}$ and $T_{3}$. In this case, let $T_{4}$ be the time this second large component is completed. (If no such second large component is found, let for definiteness $T_{3}=T_{4}=T_{2}$.)

Note that $0 \leq \widetilde{V}_{1}(t)-V_{1}(t) \leq \widetilde{S}(t)-S(t)$ for all $t$. Hence, using Lemma 6.3 and (6.8) with $T=T_{2} / \alpha_{n}$, the number of vertices of degree 1 found between $T_{2}$ and $T_{3}$ is

$$
\begin{aligned}
V_{1}\left(T_{2}-\right)-V_{1}\left(T_{3}-\right) & \geq \widetilde{V}_{1}\left(T_{2}-\right)-\left(\widetilde{S}\left(T_{2}-\right)-S\left(T_{2}-\right)\right)-\widetilde{V}_{1}\left(T_{3}-\right) \\
& =n_{1} e^{-T_{2}}-n_{1} e^{-T_{3}}+o_{\mathrm{p}}\left(n \alpha_{n}^{2}\right) .
\end{aligned}
$$

Since this is at most $n \alpha_{n}^{\prime}=o\left(n \alpha_{n}\right)$, and $n_{1} / n \rightarrow p_{1}>0$, it follows that $e^{-T_{2}}-e^{-T_{3}}=o_{\mathrm{p}}\left(\alpha_{n}\right)$, and thus $T_{3}=T_{2}+o_{\mathrm{p}}\left(\alpha_{n}\right)=\tau \alpha_{n}+o_{\mathrm{p}}\left(\alpha_{n}\right)$. Hence, (6.8) applies to $T=T_{3} / \alpha_{n}$, and since no $\mathrm{C} 1$ is performed between $T_{3}$ and $T_{4}$,

$$
\sup _{t \leq T_{4}}|\widetilde{S}(t)-S(t)| \leq \sup _{t \leq T_{3}}|\widetilde{S}(t)-S(t)|+d_{\max }=o_{\mathrm{p}}\left(n \alpha_{n}^{2}\right) .
$$

Let $t_{0}>\tau$; thus $\widetilde{H}\left(t_{0}\right)=t_{0}-\frac{1}{2} \beta t_{0}^{2}<0$ and (6.7) yields, with $\delta=$ $\left|\widetilde{H}\left(t_{0}\right)\right| / 2>0$, whp $\widetilde{A}\left(\alpha_{n} t_{0}\right) \leq-n \alpha_{n}^{2} \delta$ and thus

$$
\widetilde{S}\left(\alpha_{n} t_{0}\right)-S\left(\alpha_{n} t_{0}\right)=A\left(\alpha_{n} t_{0}\right)-\widetilde{A}\left(\alpha_{n} t_{0}\right) \geq n \alpha_{n}^{2} \delta .
$$


Hence (6.13) shows that whp $T_{4}<\alpha_{n} t_{0}$. Since $t_{0}>\tau$ is arbitrary, and further $T_{2} \leq T_{3} \leq T_{4}$ and $T_{2} / \alpha_{n} \stackrel{\mathrm{p}}{\longrightarrow} \tau$, it follows that $T_{3} / \alpha_{n} \stackrel{\mathrm{p}}{\longrightarrow} \tau$ and $T_{4} / \alpha_{n} \stackrel{\mathrm{p}}{\longrightarrow} \tau$.

Finally, by Lemma 6.4 again, this time applied to $T_{3}$ and $T_{4}$, the number of edges found between $T_{3}$ and $T_{4}$ is $o_{\mathrm{p}}\left(n \alpha_{n}\right)=o_{\mathrm{p}}\left(m \alpha_{n}\right)$. Hence, whp there is no large component found there, although the construction gave a large component with probability at least $\varepsilon_{1} \mathbb{P}\left(\mathcal{E}_{\varepsilon}\right)+o(1)$. Consequently, $\varepsilon_{1} \mathbb{P}\left(\mathcal{E}_{\varepsilon}\right)=o(1)$ and $\mathbb{P}\left(\mathcal{E}_{\varepsilon}\right)=o(1)$.

Recalling the definition of $\mathcal{E}_{\varepsilon}$, we see that whp the total number of edges in large components is at most $(2 \tau+2 \varepsilon) m \alpha_{n}$; since whp at least $(2 \tau-\varepsilon) m \alpha_{n}$ of these belong to $\mathcal{C}^{\prime}$, there are at most $3 \varepsilon m \alpha_{n}$ edges, and therefore at most $3 \varepsilon m \alpha_{n}+1$ vertices, in any other component.

Choosing $\varepsilon$ small enough, this shows that whp $\mathcal{C}_{1}=\mathcal{C}^{\prime}$, and further $v\left(\mathcal{C}_{2}\right) \leq e\left(\mathcal{C}_{2}\right)+1 \leq 3 \varepsilon m \alpha_{n}+1<3 \lambda \varepsilon n \alpha_{n}$.

\section{Conceivable extensions}

It seems to be possible to obtain quantitative versions of our results, such as a central limit theorem for the size of the giant component, as we did for the $k$-core in [16]. (See Pittel [27] and Barraez, Boucheron and de la Vega [3] for $G(n, p)$ and $G(n, m)$, and [20] for the random cluster model.) Similarly, it should be possible to obtain large deviation estimates.

Further, in the transition window, where $\alpha_{n}=O\left(n^{1 / 3}\right)$, an appropriate scaling seems to lead to convergence to Gaussian processes resembling the one studied by Aldous [1], and it seems likely that similar results on the distribution of the sizes of the largest components could be obtained.

We have not attempted above to give more precise bounds on the size of the second component $\mathcal{C}_{2}$, and we leave it as an open problem to see whether our methods can lead to new insights for this problem. It appears that direct analysis of the Markov process $\left(A(t), V_{0}(t), V_{1}(t), \ldots\right)$ can show that the largest component has size $O(\log n)$ in the subcritical phase, and that so does the second largest component in the supercritical case, but we have not pursued this. Such a result would imply a type of phase transition very much like that known to occur in $G(n, p)$ and certain other types of graphs, such as the Hamming graph $H(2, n)$ (see [21]).

Finally, it seems possible to adapt the methods of this paper to random hypergraphs and obtain results similar to those in Behrisch, Coja-Oghlan and Kang [4], but we leave this to the reader.

Acknowledgements. This research was partly done during a visit by SJ to the University of Cambridge, partly funded by Trinity College. MJL was partly supported by the Nuffield Foundation and by a Research Fellowship from the Humboldt Foundation. 


\section{REFERENCES}

[1] D. Aldous, Brownian excursions, critical random graphs and the multiplicative coalescent. Ann. Probab. 25 (1997), 812-854.

[2] R. Arratia, A.D. Barbour and S. Tavaré, Logarithmic Combinatorial Structures: a Probabilistic Approach, EMS, Zürich, 2003.

[3] D. Barraez, S. Boucheron and W. Fernandez de la Vega, On the fluctuations of the giant component. Combin. Probab. Comput. 9 (2000), 287-304.

[4] M. Behrisch, A. Coja-Oghlan and M. Kang, Local limit theorems and the number of connected hypergraphs. Preprint, 2007. arXiv:0706.0497v1

[5] B. Bollobás, Random Graphs, 2nd ed., Cambridge Univ. Press, Cambridge, 2001.

[6] B. Bollobás, S. Janson \& O. Riordan, The phase transition in inhomogeneous random graphs. Random Struct. Alg. 31, 3-122.

[7] C. Borgs, J. Chayes, R. van der Hofstad, G. Slade and J. Spencer, Random subgraphs of finite graphs: III. The phase transition for the n-cube. Combinatorica 26 (2006), 395-410.

[8] T. Britton, S. Janson \& A. Martin-Löf, Graphs with specified degree distributions, simple epidemics and local vaccination strategies. Advances Appl. Probab. 39 (2007), no. 4, 922-948.

[9] T. Britton, M. Deijfen \& A. Martin-Löf, Generating simple random graphs with prescribed degree distribution, J. Statist. Phys., to appear.

[10] G. Grimmett \& S. Janson, Random graphs with forbidden vertex degrees. Preprint, 2007. arXiv:0712.0270v1

[11] A. Gut, Probability: A Graduate Course. Springer, New York, 2005.

[12] R. van der Hofstad and M.J. Luczak, Random subgraphs of the 2D Hamming graph: the supercritical phase, Probab. Theor. Relat. Fields, to appear. arXiv:0801.1607

[13] S. Janson, The probability that a random multigraph is simple. Preprint, 2006. arXiv:math.C0/0609802

[14] S. Janson, D.E. Knuth, T. Euczak \& B. Pittel, The birth of the giant component, Random Struct. Alg. 3 (1993), 233-358.

[15] S. Janson and M. Luczak, A simple solution to the $k$-core problem. Random Struct. Alg. 30 (2007), no. 1-2, 50-62.

[16] S. Janson \& M. Luczak, Asymptotic normality of the $k$-core in random graphs. Ann. Appl. Probab., to appear. arXiv:math.C0/0612827

[17] S. Janson, T. Łuczak \& A. Ruciński, Random Graphs, Wiley, New York, 2000.

[18] O. Kallenberg, Foundations of Modern Probability, 2nd ed., Springer, New York, 2002.

[19] M. Kang and T.G. Seierstad, The critical phase for random graphs with a given degree sequence. Combin., Probab., Comput., to appear. 
[20] M. Luczak and T. Łuczak, The phase transition in the cluster-scaled model of a random graph. Random Struct. Alg. 28 (2006), 215-246.

[21] M. Luczak and J. Spencer, The second component in the supercritical 2D Hamming graph. Preprint, 2007. arXiv:0801.1608

[22] B. D. McKay, Asymptotics for symmetric 0-1 matrices with prescribed row sums. Ars Combin. 19A (1985), 15-25.

[23] B. D. McKay \& N. C. Wormald, Asymptotic enumeration by degree sequence of graphs with degrees $o\left(n^{1 / 2}\right)$. Combinatorica 11 (1991), no. $4,369-382$.

[24] M. Molloy \& B. Reed, A critical point for random graphs with a given degree sequence, Random Struct. Alg. 6 (1995), no. 2-3, 161-179.

[25] M. Molloy \& B. Reed, The size of the largest component of a random graph on a fixed degree sequence, Combin. Probab. Comput. 7 (1998), 295-306.

[26] A. Nachmias and Y. Peres, Critical percolation on random regular graphs. Preprint, 2007. arXiv:0707.2839v2

[27] B. Pittel, On tree census and the giant component in sparse random graphs. Random Struct. Alg. 1 (1990), 311-342.

Department of Mathematics, Uppsala University, PO Box 480, SE-751 06 UPPSALA, SWEDEN

E-mail address: svante.janson@math.uu.se

URL: http://www.math.uu.se/ ${ }^{\sim}$ svante/

Department of Mathematics, London School of Economics, Houghton Street, LONDON WC2A 2AE, United Kingdom

E-mail address: malwina@planck.lse.ac.uk

$U R L:$ http://www.lse.ac.uk/people/m.j.luczak@lse.ac.uk/ 